\title{
FSC-Q: A method for quality analysis of cryoEM-derived models
}

\section{Erney Ramírez-Aportela, David Maluenda, Yunior C. Fonseca, Pablo Conesa, Roberto Marabini, Carlos Oscar S. Sorzano and Jose Maria Carazo}

\author{
Biocomputing Unit, National Center for Biotechnology (CSIC), Darwin 3, Campus Univ. Autónoma de Madrid, Cantoblanco, 28049 \\ Madrid, Spain \\ erney.ramirez@gmail.com
}

To obtain more accurate atomic models from cryoEM and increase their impact on biomedical research, metrics are needed that carefully evaluate these constructed models. In this poster we present further developments on FSC-Q, a map-to-model quality issue recently introduced [1], with the capability to detect those areas of the model that are better supported by the experimental data (Figure1). The algorithm performs a careful analysis of the Signal-to-Noise Ratio in the half maps and in map generated from the proposed model through local resolution. It is intuitive and, yet, very precise, introducing quality information that we have quantitatively shown is new, in the sense that some of it was not captured in previous quality assessment metrics.

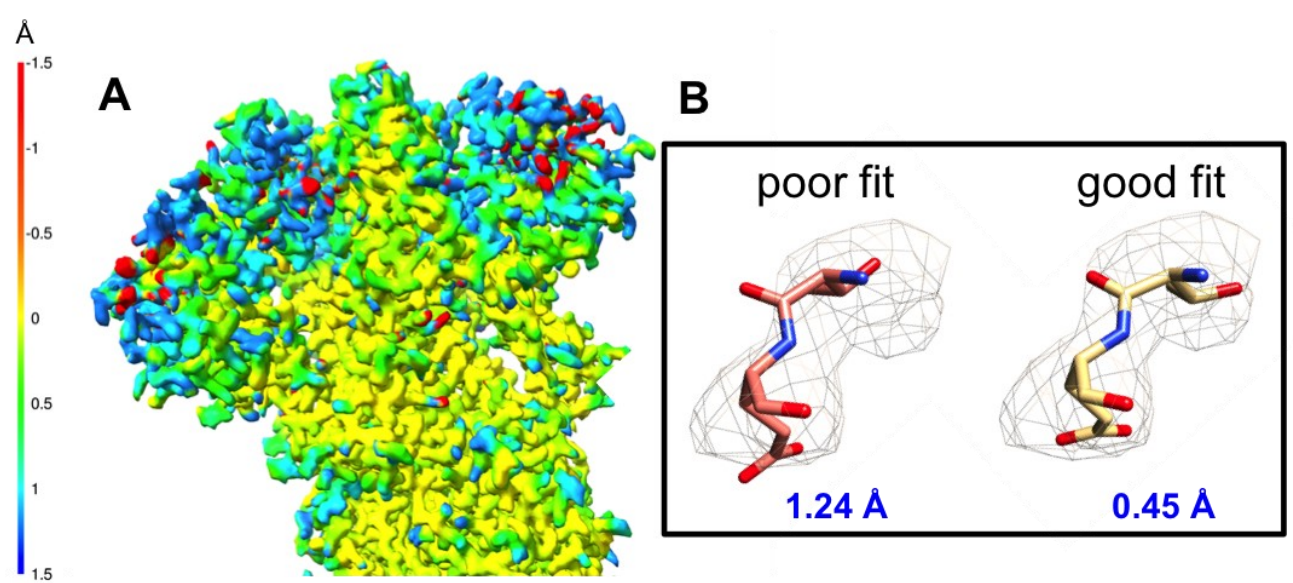

Figure 1: Structure-fit analysis of the closed-state SARS-CoV-2 spike glycoprotein (emd-21452) using FSC-Q. A) FSC-Q are represented on the map generated from the atomic model (PDB: id-6vxx). Scale: red indicates atoms that may be associated with noise and blue indicates poorly fitted atoms or areas with low resolvability. B) The panel shows the original fit (left) of amino acids THR-323 and GLU-324 and the improved fit (right) using ISOLDE [2]. Under each fragment, the average FSC-Q is shown.

[1] FSC-Q: a CryoEM map-to-atomic model quality validation based on the local Fourier shell correlation. Ramírez-Aportela E, Maluenda D, Fonseca YC, Conesa P, Marabini R, Heymann JB, Carazo JM, Sorzano COS.Nat Commun. 2021 Jan 4;12(1):42.

[2] ISOLDE: a physically realistic environment for model building into low-resolution electron-density maps. Croll, T. I. Acta Crystallogr. D Struct. Biol. 74, 519-530 (2018).

Keywords: CryoEM, map-to-model validation, local resolution 